\title{
Efeito de biofertilizante no desenvolvimento agronômico da cultura da rúcula Eruca sativa Miller cv. Folha Larga
}

\section{Edcleyton José de Lima ${ }^{1}$, , Raquel Maria da Silva ${ }^{2}$, Lucas Ferreira da Silva ${ }^{1}$, Juliete Amanda Theodora de Almeida ${ }^{3}$, Moema Kelly Nogueira de Sá $^{3}$ e Fernando Ferreira da Silva Dias ${ }^{4}$}

${ }^{1}$ Universidade Federal do Agreste de Pernambuco. Curso de Graduação em Engenharia Agronômica. Av. Bom Pastor, S/№. Boa Vista. Garanhuns-PE, Brasil (CEP 55292-270). E-mail: cley1020kj@gmail.com.

${ }^{2}$ Universidade Federal do Agreste de Pernambuco. Programa de Pós-Graduação em Produção Agrícola. Av. Bom Pastor, S/№. Boa Vista. Garanhuns-PE, Brasil (CEP 55292-270).

${ }^{3}$ Universidade Federal do Agreste de Pernambuco. Programa de Pós-Graduação em Ciência Animal e Pastagens. Av. Bom Pastor, S/№. Boa Vista. Garanhuns-PE, Brasil (CEP 55292-270).

${ }^{4}$ Universidade Federal do Agreste de Pernambuco. Av. Bom Pastor, S/№. Boa Vista. Garanhuns-PE, Brasil (CEP 55292-270).

Resumo. A busca por alimentos livres de substâncias químicas fez com que a agricultura orgânica atingisse patamares mundiais, dessa forma, novas tecnologias e métodos que possibilitem a inovação da agricultura orgânica e sustentável, são amplamente estudados. Buscando diversificar os biofertilizantes alternativos nas hortícolas em detrimento ao uso desenfreado de adubos industriais, essa pesquisa teve como objetivo avaliar doses percentuais de biofertilizante de esterco bovino na cultura da rúcula Eruca sativa Miller cv. Folha Larga. A pesquisa foi realizada no período de maio a junho de 2019 em casa de vegetação, localizada na área experimental da Universidade Federal do Agreste de Pernambuco (UFAPE). Foi utilizado o delineamento experimental inteiramente casualizado (DIC), com cinco tratamentos e cinco repetições. Os tratamentos referem-se as concentrações do biofertilizante $0 \%, 5 \%, 10 \%, 15 \%$ e $20 \% \cdot \mathrm{L}^{-1}$, aplicados em dose única, sendo a dose $0 \%$ considerada a testemunha negativa aplicada com $\mathrm{H}_{2} \mathrm{O}$ destilada, também foram quantificados o pH e o teor de sólidos do biofertilizante utilizado. Os parâmetros avaliados foram: número de folhas (NF), comprimento da raiz (CR) e altura da planta (AP). 0 teste de Tukey a $5 \%$ de probabilidade, foi adotado para comparar as variâncias de populações normais independentes. 0 teor de sólidos encontrados estavam próximo de 0 , estando dentro dos valores recomendados, o biofertilizante utilizado tinha $\mathrm{pH}$ de 7,2 .

Recebido

$26 / 02 / 2021$

Aceito

$25 / 04 / 2021$

Publicado

$30 / 04 / 2021$

Acesso aberto

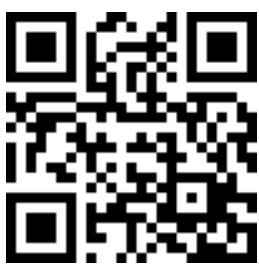

0000-0002-3635-1369

Edcleyton José de Lima

ISSN 2359-1412/RBGAS-2021-0020/2021/8/18/36/559

Rev. Bras. Gest. Amb. Sustent.

http://revista.ecogestaobrasil.net 
Quanto as concentrações a de $20 \%$ foi o que apresentou menor quantitativo de NF, CR e AP, já a dose de $5 \%$ apresentou os melhores resultados para tais parâmetros, porém sem diferença estatística em relação as doses de $10 \%$ e $15 \%$. Constata-se que a dose de 5\% é a mais recomendada nas presentes condições para o plantio da cultivar. Devido ao crescimento potencial e econômico da rúcula no mercado de hortaliças, conclui-se que novas pesquisas com adubos não industriais devem ser realizadas com intuito de ofertar alternativas viáveis de biofertilizantes para uso em cultivo orgânico.

Palavras-chaves: Cultivo orgânico; Hortícolas; Adubo orgânico.

Abstract. Effect of biofertilizer on the agronomic development of the arugula crop Eruca sativa Miller cv. Broad Leaf. The search for chemical-free foods has led organic agriculture to reach global levels, thus, new technologies and methods that enable the innovation of organic and sustainable agriculture are widely studied. Seeking to diversify alternative biofertilizers in vegetables to the detriment of the rampant use of industrial fertilizers, this research aimed to evaluate percentage doses of biofertilizer of bovine manure in the arugula Eruca sativa Miller culture (cultivar Folha Larga). The research was carried out from May to June 2019 in a greenhouse, located in the experimental area of the Federal University of Agreste de Pernambuco (UFAPE). A completely randomized design (DIC) was used, with five treatments and five replications. The treatments refer to the concentrations of biofertilizer, at $0 \%, 5 \%, 10 \%, 15 \%$ and $20 \% . \mathrm{L}^{-1}$, applied in a single dose, with the $0 \%$ dose being considered the negative control applied with distilled $\mathrm{H}_{2} \mathrm{O}$, were also quantified the $\mathrm{pH}$ and the solids content of the biofertilizer used. The evaluated parameters were: number of leaves (NF), root length (CR) and plant height (AP). The Tukey test at 5\% probability was adopted to compare the variances of independent normal populations. The solids content found were close to 0 , being within the recommended values, the biofertilizer used had a $\mathrm{pH}$ of 7.2. As for the concentrations at $20 \%$, it was the one with the lowest amount of NF, CR and AP, whereas the dose of $5 \%$ showed the best results for such parameters, but without statistical difference in relation to the doses of $10 \%$ and $15 \%$. It appears that the $5 \%$ dose is the most recommended in the present conditions for planting the cultivar. Due to the potential and economic growth of arugula in the vegetable market, it is concluded that further research with non-industrial fertilizers should be carried out in order to offer viable alternatives to biofertilizers for use in organic cultivation.

Keywords: Organic cultivation; Vegetables; Organic fertilizer.
D) 0000-0002-5503-2072

Raquel Maria da Silva

(1) 0000-0002-7913-5500

Lucas Ferreira da Silva

(1) 0000-0002-5787-6543

Juliete Amanda

Theodora de Almeida

D 0000-0001-5748-709X

Moema Kelly de Sá

(c) 0000-0002-8292-683X

Fernando Ferreira da

Silva Dias 


\section{Introdução}

A busca por alimentos livres de substâncias químicas fez com que a agricultura orgânica atingisse patamares mundiais, culminando no seu registro e incentivo em mais de 150 países, incluindo grandes potencias, como Estados Unidos, países europeus, Japão, dentre outros. Tal incentivo é visto como uma forma de minimizar os efeitos danosos de agrotóxicos que são lançados no meio ambiente e nos alimentos (Santos et al. 2012).

Alimentos de origem orgânica compreendem os seguintes requisitos: não utilização de agrotóxicos, técnicas de cultivos que mantenham o ciclo de elementos naturais, adubação verde, rotação de culturas, preservação do solo e da biodiversidade, entre diversos outros fatores (Pacífico, 2017). A adubação é um dos principais entraves para a sustentabilidade de cultivos, pois elementos como o fósforo, nitrogênio e potássio são extraídos de fontes não renováveis (Ziesemer, 2007).

Uma das formas de substituir a adubação convencional, situa-se no uso de biofertilizantes de origem animal ou vegetal, pois além dos macronutrientes como potássio, fósforo, magnésio e nitrogênio, há também a presença de micronutrientes (Mendonça et al., 2016). Há inúmeras vantagens no uso de biofertilizante, entre elas estão: reciclagem dos nutrientes, eleva o $\mathrm{pH}$ e contribui para melhorar as características físicas e químicas do solo (Alencar et al., 2015).

As hortaliças estão entre os alimentos com maior quantitativo de substâncias tóxicas, devido ao grande quantitativo de pragas e doenças que as acometem e o manejo tradicional atualmente empregado (Silva, 2012; Reitz et al., 2013). 0 cultivo de hortaliças orgânicas vem crescendo gradativamente, pois além de questões sociais e de sanidade dos consumidores, outro aspecto importante é o baixo custo, porém ainda há um déficit de pesquisas e conhecimentos técnicos para expansão dessa prática para muitas culturas de interesse agrícola no Brasil (Almeida e Junqueira, 2011).

A rúcula Eruca sativa Miller é uma hortaliça herbácea pertencente à família Brassicaceae, apresenta curto período de ciclo, possui teores significativos de enxofre, ferro, cálcio e vitamina A e C (Figueiredo et al., 2007; Salles et al., 2018). Seu alto valor nutricional e seu sabor picante, faz com que suas folhas sejam utilizadas na culinária, principalmente nas regiões sul, sudeste e nordeste (Silva et al., 2012). Devido a essas características essa folhosa vem apresentando crescimento expressivo no consumo, mostrando ser um mercado com potencial promissor (Oliveira et al., 2018).

Entre as hortaliças com maior quantitativo de estudos com sistemas orgânicos está a rúcula, pois seu cultivo é de ciclo curto e seu mercado vem crescendo no Brasil, desta forma, estudos com viabilidade técnico-científica devem ser aprimorados e incentivados (Steiner et al., 2012). Assim, neste estudo, testamos a validade da hipótese de que a utilização de biofertilizante de esterco bovino aplicado via solo irá proporcionar uma maior produtividade. Desta forma, esta pesquisa objetivou verificar qual a dose de biofertilizante assim como a sua utilização na cultura sobe condições experimentais controladas.

\section{Metodologia}

A pesquisa foi realizada no período de maio a junho de 2019 em casa de vegetação, localizada na área experimental da Universidade Federal do Agreste de Pernambuco (UFAPE) com coordenadas geográficas $08^{\circ} 53^{\prime} 25^{\prime \prime} \mathrm{S}$ e $36^{\circ} 29^{\prime} 34^{\prime \prime} 0$, a altitude média é de $896 \mathrm{~m}$ com temperatura média anual de $21{ }^{\circ} \mathrm{C}$, sendo os meses mais chuvosos: maio, junho, julho e agosto (Canuto et al., 2019). 0 biofertilizante utilizado foi proveniente do biorreator do Laboratório Multiusuário de Meio Ambiente/UFAPE e tem como ingredientes água e esterco bovino. 
Foram coletadas amostras do biofertilizante e verificado o $\mathrm{pH}$ juntamente com uma fração de nove sólidos, sendo eles sólidos totais (ST), sólidos dissolvidos totais (SDT), sólidos suspensos totais (SST), sólidos fixos totais (SFT), sólidos voláteis totais (SVT), sólidos dissolvidos fixos (SDF), sólidos dissolvidos voláteis (SDV), sólidos suspensos fixos (SSF) e sólidos suspensos voláteis (SSV).

Foi utilizado o delineamento experimental inteiramente casualizado, com cinco tratamentos e cinco repetições (Figura 1). Os tratamentos referem-se às concentrações do biofertilizante $\left(0 \%, 5 \%, 10 \%, 15 \%\right.$ e $\left.20 \% \mathrm{~L}^{-1}\right)$, aplicados em dose única, sendo a dose $0 \%$ testemunha negativa aplicada com $\mathrm{H}_{2} \mathrm{O}$ destilada.

A cultivar de rúcula empregada foi a Folha Larga, sementes apresentando uma germinação de $95 \%$ e pureza física de 99,9\%. A semeadura da rúcula foi realizada diretamente no solo, sendo três sementes por recipiente, após sete dias foi realizado o desbaste, mantendo-se uma planta, ao total foram utilizadas 25 unidades experimentais para a avaliação dos resultados.

Para o plantio das sementes de rúcula, foram utilizadas porções de areia lavada seguindo a metodologia de Medeiros et al. (2007), e posteriormente foi submetida a lavagem com água destilada em fluxo corrente para retirada das impurezas, os recipientes receberam a capacidade máxima de substrato para o seu volume.

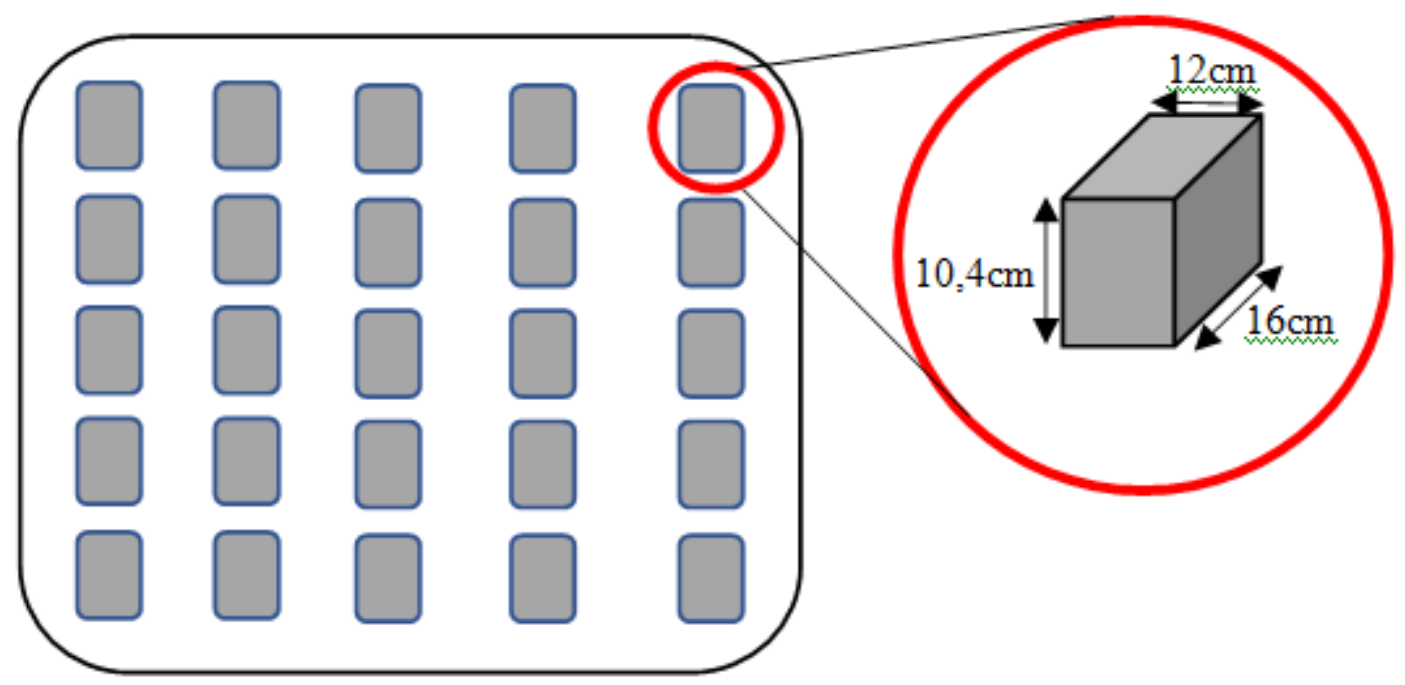

Figura 1. Sistema de distribuição experimental de cultivo.

As plantas foram irrigadas uma vez ao dia, com base no valor de capacidade de campo (CC) de $0,135 \mathrm{~cm}^{3} \cdot \mathrm{cm}^{-3}$, que é o conteúdo de água necessário para manter o solo na CC. A colheita da rúcula foi realizada 47 dias após a emergência, onde foram realizadas as avaliações do número de folhas (NF), comprimento da raiz (CR) e altura da planta (AP).

Os dados foram submetidos a testes de normalidade e homogeneidade, onde foram realizadas as análises de variância, adotando-se o "teste de Tukey", para comparar as variâncias de populações normais independentes. 


\section{Resultados e discussão}

Os teores de sólidos analisados no biofertilizante apresentaram resultados satisfatórios, onde foram encontrados inicialmente valores abaixo de $1 \%$ em todas as nove frações e após 30 dias de funcionamento do biodigestor, a presença de sólidos no substrato resultante da fermentação foi próxima de zero, sendo assim, indicando o sucesso na biodigestão. Em estudos com produção de biogás, Bulhões e Mussolini (2016) explicitam que a diminuição no teor de sólidos resulta em maior produção de biogás e, consequentemente, não ocorre a deposição de dejetos não fermentados no solo.

0 biofertilizante utilizado tinha $\mathrm{pH}$ de 7.2 , ou seja, com caráter neutro, não apresentava odor forte e característico do esterco bovino, indicando que sua fermentação anaeróbica ocorreu de maneira adequada. 0 composto oriundo do reator anaeróbico, proporciona, segundo Oliveira et al. (2019), a recuperação e mantimento da microbiota do solo, estimulação do crescimento e desenvolvimento de plântulas e o fortalecimento do sistema de defesa das plantas frente ao ataque de pragas e doenças.

0 crescimento das plantas por meio da utilização de biofertilizante é notório, principalmente na aplicação de uma dose que atenda às necessidades nutricionais da planta, Silva et al. (2019) afirmam que o uso também contribui para desenvolvimento de uma agricultura sustentável, sendo importante para a manutenção da qualidade dos cultivos.

No presente estudo foi verificado maior crescimento de mudas nos tratamentos que receberam o biofertilizante, sendo a dose de $5 \%$ a que apresentou o maior resultado, porém estatisticamente igual aos demais. Em estudos com alface Lactuca sativa L. cv. Isabel, Santos et al. (2021), pesquisando os efeitos com compostos orgânicos em mudas, constataram maior produção dessa hortícola, avaliando o tamanho da cabeça e a massa foliar com uso de composto orgânico e biofertilizante. Nomura et al. (2019), avaliando concentrações de biofertilizante e adubação química NPK 20-0-20, verificaram que a química não diferenciou no parâmetro massa seca de uma diluição intermediária de $0,5 \mathrm{~mL} . \mathrm{L}^{-1}$ do biofertilizante.

De acordo com os resultados obtidos, foi possível verificar que, em relação ao número de folhas (NF) os tratamentos apresentaram efeito significativo. A concentração de $20 \%$ de biofertilizante apresentou baixa média no número de folhas por planta (5 folhas.planta-1) em relação à concentração de $5 \%$, mas não havendo diferença de médias em comparação aos demais tratamentos. Matos et al. (2016), analisando um biofertilizante de esterco bovino com na concentração de 136,5 kg de N.ha- ${ }^{-1}$, obtiveram produção média de 8,3 folhas. Os tratamentos com concentrações de $0 \%, 10 \%$ e 15\% não diferiram de nenhum dos tratamentos ao nível de $5 \%$ de significância (Tabela 1).

Tabela 1. Número de folhas (folhas.planta ${ }^{-1}$ ), altura de planta (cm.planta-1) e comprimento de raiz (cm.planta- ${ }^{-1}$ ).

\begin{tabular}{|c|c|c|c|}
\hline Doses de biofertilizantes (\%) & Número de folhas & Altura de planta & Comprimento de Raiz \\
\hline 0 & $9,4 \mathrm{ab}$ & $23,46 \mathrm{a}$ & $9,34 \mathrm{a}$ \\
\hline 5 & $10,4 \mathrm{a}$ & $24,68 \mathrm{a}$ & $11,56 \mathrm{a}$ \\
\hline 10 & $9,2 \mathrm{ab}$ & $23,52 \mathrm{a}$ & $10,06 \mathrm{a}$ \\
\hline 15 & $9,4 \mathrm{ab}$ & $19,94 \mathrm{a}$ & $10,02 \mathrm{a}$ \\
\hline 20 & $5 \mathrm{~b}$ & $8,8 \mathrm{~b}$ & $5,8 \mathrm{a}$ \\
\hline
\end{tabular}

*Médias seguidas de mesma letra minúscula na coluna não diferem estatisticamente a $5 \%$ probabilidade pelo teste de Tukey. 
Quanto à variável altura de planta (AP) a média dos tratamentos teve diferença significativa, onde a que recebeu maior quantitativo de biofertilizante, obteve menor média, as demais foram estatisticamente iguais, Santos e Akiba (1996) afirmam que em altas concentrações os biofertilizantes podem acarretar em desvios metabólicos que estão ligados à produção de substâncias de defesa, impactando no atraso do crescimento, floração ou frutificação de muitas espécies vegetais. Em estudos avaliando torta de filtro, torta de nim, húmus de minhoca e um biofertilizante cujo ingrediente também foi esterco em condições de campo, Matos et al. (2016), concluíram que as plantas de rúcula que receberam o biofertilizante na dose correta apresentaram melhor desempenho, refletindo em uma AP de $17,1 \mathrm{~cm}$.

Quanto ao parâmetro comprimento da raiz, o tratamento que diferenciou e obteve a menor média foi com biofertilizante a $20 \%$. Os demais tratamentos apresentaram valores estatisticamente iguais, porém o tratamento com $5 \%$ apresentou maior valor de média. Em estudos com rúcula cultivada com adubos orgânicos e industriais, Maia et al. (2007) verificaram melhor crescimento da cultivar Folha Larga somente com adubo orgânico. Em estudos com efluentes bovino provenientes de um biodigestor, Morais (2019) obteve melhor média de crescimento no comprimento da raiz de mudas de rúcula com biofertilizante a $5 \%$, corroborando com os valores encontrados nessa pesquisa.

\section{Conclusão}

Esta pesquisa demonstrou que houve melhor desenvolvimento das variáveis avaliadas com a utilização do biofertilizante de esterco bovino a $5 \% \cdot \mathrm{L}^{-1}$, sendo a melhor dose recomendada para o plantio de mudas de rúcula da variedade Folha Larga. A dose de $20 \% . \mathrm{L}^{-1}$ resultou nos menores quantitativos das variáveis, sendo considerada uma dose prejudicial a esta planta.

Devido ao seu rápido crescimento potencial de mercado e a possibilidade de expansão do seu cultivo utilizando fertilizantes não comerciais, conclui-se que novas pesquisas devem ser realizadas com intuito de possibilitar cada vez mais o cultivo otimizado dessa hortícola.

\section{Conflito de interesses}

Os autores declaram não haver conflito de interesses.

\section{Referências}

Alencar, T. L.; Chaves, A. F.; Santos, C. L. A.; Assis Júnior, R. M.; Mota, J. C. A. Atributos físicos de um Cambissolo cultivado e tratado com biofertilizante na Chapada do Apodi. Revista Brasileira de Ciência do Solo, v. 39, n. 3, p. 737-749, 2015. https://doi.org/10.1590/ 01000683rbcs20140437

Almeida, I. L.; Junqueira, A. M. R. Produção de hortaliças em sistema orgânico, agricultura familiar e segurança alimentar no Brasil. Sociedade e Desenvolvimento Rural online, v. 5, n. 1, p. 1-15, 2011.

Canuto, C.; Andrade, D. S.; Lima, E. M.; Goes, M. C. C.; Almeida, M. M. V; Lima, J. R. S. Biochar e esterco bovino aumentam à eficiência no uso de água da alface. Diversitas Journal, v. 4, n. 3, p. 1084, 2019. https://doi.org/10.17648/diversitas-journal-v4i3.822 
Figueiredo, B. T.; Figueiredo, R. T.; Guiscem, J. M.; Araújo, A. M. S. C. J. R. G.; Pereira, C. F. M.; Farias, A. S. Produção de rúcula (Eruca sativa L.) cultivada em composto de estercos de ave e bovino puros e incorporados ao solo. Revista Brasileira de Agroecologia, v. 2, n. 2, p. 851-854, 2007.

Maia, A. F. C. A.; Medeiros, D. C.; Liberalino Filho, J. Adubação orgânica em diferentes substratos na produção de mudas rúcula. Revista Verde de Agroecologia e Desenvolvimento Sustentável, v. 2, n. 2, p. 1-7, 2007.

Matos, E. R.; Santos, B.C.; Costa, A. R.; Silva, P. C. Uso de compostos agroindustriais como biofertilizantes na produção de rúcula. Anais da 10 ${ }^{\mathrm{a}}$ Jornada Acadêmica da Jornada da UEG - Integrando Saberes e Construindo Conhecimento, v. 10, n. 1, p. 1-8, 2016. Disponível em: <https://www.anais.ueg.br/index.php/jaueg/article/view/7311>. Acesso em: 04 mar. 2021.

Medeiros, D. C.; Lima, B. A. B.; Barbosa, M. R.; Anjos, R. S. B.; Borges, R. D.; Cavalcante Neto, J. G.; Marques, L. F. Produção de mudas de alface com biofertilizantes e substratos. Horticultura Brasileira, v. 2, n. 3, p. 433-436, 2007. https://doi.org/10.1590/S010205362007000300021

Mendonça, H. V.; Ometto, Rocha, W. S. D.; Martins, C. E.; Otenio, M. H.; Borges, C. A. V. Crescimento de cana-de-açúcar sob aplicação de biofertilizante da bovinocultura e ureia. Revista Agronegócio e Meio Ambiente, v. 9, n. 4, p.973-998, 2016. https://doi.org/10.17765/2176-9168.2016v9n4p973-987

Morais, V. P. Efluentes de biodigestor e psicultura na produção de rúcula. Chapadinha: Universidade Federal do Maranhão, 2019. (Monografia).

Nomura, M.; Barbosa, G. G. F.; Silva, C. H. L.; Costa, E. M.; Ventura, M. V. A.; Vilarinho, M. S.; Pereira, L. S. Biofertilizante na produção de rúcula. Ipê Agronomic Journal, v. 3, n. 1, p. 116-121, 2019.

Oliveira, R. C.; Silva, J. E. R.; Aguilar, A. S.; Peres, P.; Luz, J. M. Q. Uso de fertilizante organomineral no desenvolvimento de mudas de rúcula. Agropecuária Científica no Semiárido, v. 14, n. 1, p. 1-6, 2018.

Pacífico, V. Alimentos orgânicos: benefícios para a saúde, meio ambiente e comércio. Revista Nawa, v. 1, n. 1, p. 1-5, 2017.

Reitz, S. R.; Gao, Y.; Lei, Z. Insecticide use and the ecology of Liriomyza leafminer management. In: Trdan, S. Insecticide: Development of safer and more effective technologie. London: Intechopen, 2013. p. 234-253. https://doi.org/10.5772/53874

Salles, J. S.; Steiner, F.; Abaker, J. E. P.; Ferreira, T. S.; Martins, G. L. M. Resposta da rúcula à adubação orgânica com diferentes compostos orgânicos. Revista de Agricultura Neotropical, v. 4, n. 2, p. 35-40, 2017.

Santos, A. C. V.; Akiba, F. Biofertilizante líquido: uso correto na agricultura alternativa. Seropédica: UFRRJ, Imprensa Universitária, 1996.

Santos, J. O.; Santos, R. M. S.; Borges, M. G. B.; Ferreira, R. T. F. V.; Salgado, A. B.; Santos Segundo, 0. A. A evolução da agricultura orgânica. Revista Brasileira de Gestão Ambiental, v. 6, n. 1, p. 35-41, 2012.

Silva, A. O.; Soares, T. M.; Silva, E. F. F.; Santos, A. N.; Klar, A. E. Consumo hídrico da rúcula em cultivo hidropônico NFT utilizando rejeitos de dessalinizador em Ibimirim-PE. Irriga, v. 17, n. 1, p. 114-125, 2012. 
Silva, C. F. A.; Lattini, A. O.; Lofrano, R. C. Z. Efeito de biofertilizante no crescimento de alface, rúcula, tomate, cebolinha e repolho. Revista Gestão \& Sustentabilidade Ambiental, v. 8, n. 3, p. 278-287, 2019. https://doi.org/10.19177/rgsa.v8e32019278-287

Silva, R. F. Novo manual de olericultura: agrotecnologia moderna na produção e comercialização de hortaliças. 2. ed. Viçosa: UFV, 2012.

Steiner, F.; Echer, M. M.; Guimarães, V. F. Produção de alface 'Piraroxa' afetada pela adubação nitrogenada com fertilizante orgânico e mineral. Scientia Agraria Paranaensis, v. 11, n. 3, p. 77-83, 2012. https://doi.org/10.18188/1983-1471/sap.v11n3p77-83

Ziesemer, J. Energy use in organic food systems. Roma: FAO, 2007. 